\title{
Neutrino anomalies and chiral flipping in anisotropic Einstein-Cartan cosmology
}

\author{
By L.C. Garcia de Andrade ${ }^{1}$
}

\begin{abstract}
Recently Palle has investigated the chiral vorticity and Cartan torsion in neutrino asymmetries. In his case he addressed this problem in Goedel s like anisotropic Einstein-Catan cosmology. In this paper we discusse how these ideas applied to sheared Bianchi types I EinsteinCartan (EC) neutrino amisotropic cosmology, affect the handness of neutrinos in the universe. Actually here a novel concept of the chiral metric is introduced where metric functions also possess two distinct signs as in neutrino flipping or helicity. The axial anomaly equation for neutrinos in the presence of torsion and metric chirality is shown to produce left-handed neutrinos from right-handed torsion. Metric chirality is shown to be able to define how the metric would behave far away of neutrino density. Chiral flipping of the chiral neutrinos in the presence of torsion is also investigated. It is shown that when the chiral torsion is left-handed the chemical chiral potential vanishes as the universe expands.
\end{abstract}

\footnotetext{
${ }^{1}$ Departamento de Física Teórica - IF - UERJ - Rua São Francisco Xavier 524, Rio de Janeiro, RJ, Maracanã, CEP:20550. e-mail:luizandra795@gmail.com
} 


\section{Introduction}

Earlier D Palle [1] has investigated how neutrino asymmetry induces chiral vorticity and torsion handness of the universe. This task was accomplished by making use of Goedel like rotating metric in the realm of Einstein-Cartan cosmology with spin-torsion neutrino quantum helicity sources, or quantum torsion [2,3]. Palle has used Majorana neutrinos in his attempt to investigate chirally asymmetric weak interactions in anisotropic EC universes. CP violation, which has been recently solved in its strong version by Karananas [4] is also linked with torsion. Palle discovered that the universe has a righthanded vorticity chirality while neutrinos possess a left-handed chirality [1]. Recently right-handed torsionful universes have been also discovered to appear in the context of positive cosmological constant even for non-spinorial metric. Magueijo et al [3] has associated wave functions and cosmological constant to quantum torsion in Einstein-Cartan gravity extension with a Pontryagin term in the action. In isotropic FRW cosmology in EC gravity, Dolan [5] has investigate chiral fermions in this early universe with torsion. All of these works seems to lend support and motivated us to further investigate metric-torsion chirality besides chiral dynamo cosmology [6]. Chiral anomalies in astrophysical torsionful models for Black holes and gravitational waves have been developed very recently by the author, also in teleparallel geometry [7]. Chirality of the metric functions in the present paper are induced from the neutrino asymmetry helicities and solutions of anisotropic EC cosmology [8]. Neutrino oscillations in the presence of a torsion field, discovered by de Sabbata and Gasperini [9], are investigated to be connected to the metric-torsion spacetime. More recently Capozziello et al [10] has further investigate the neutrino oscillations. In their case torsion eigenstates of Hamiltonian matrices produces different mixtures of right and left-handed chiralities. Helicity flip on neutrinos can be obtained by in chiral magnetic fields as shown by Boyarsky et al [11]. In the same way it seems torsion may act as a magnetic field and cause this kind of flipping. The paper is organised as follows: In section 2 we compute the EC cosmological type-I Bianchi model with chiral asymmetric neutrinos as sources, and show that the chiral metric new concept appears naturally from the EC equations exact solutions. In section 3 the Bianchi type I solution of EC gravity is used to compute chiral flipping from torsional anomalies. De Sitter spacetime is also shown to induce left-handed neutrinos from chiral torsion from axial anomaly. Section 
4 is left for discussions and conclusions.

\section{Chiral metric from neutrino asymmetries in Bianchi type I cosmology}

Let us perform in this section the computation of EC anisotropic Bianchi type I cosmology with neutrino sources. Bianchi type I Einstein-Cartan anisotropic cosmology metric [8] can be expressed as the line element of differential geometric expression

$$
d s^{2}=d t^{2}-e^{2 \phi} d x^{2}-e^{2 \psi} d y^{2}-e^{2 \theta} d z^{2}
$$

From the usual classical non-extended EC gravity action variation

$$
\delta \int\left[R+\frac{16 \pi G}{c^{4}} L\right] \sqrt{-g} d^{4} x=0
$$

where $\mathrm{L}$ represents the Lagrangean for the non-gravitational fields. Variation of the metric $g_{i k}$, where $(i, j=0,1,2,3)$ and the torsion tensor $T^{i}{ }_{j k}$ independently lead us to obtain the classical Einstein-Cartan equations of gravity which represents a deformation of the Einstein s general relativity. In the case of anisotropic metric (1), may obtained by firstly expressing the components of Riemannian Einstein tensor as

$$
\begin{aligned}
G^{1}{ }_{1} & =-(\ddot{\psi}+\ddot{\theta})+(\dot{\psi}+\dot{\theta}+\dot{\phi}) \frac{1}{2}[\dot{\phi}-\dot{\psi}-\dot{\theta}]-\frac{1}{2}\left[\dot{\phi}^{2}+\dot{\psi}^{2}+\dot{\theta}^{2}\right] \\
G^{2}{ }_{2} & =-(\ddot{\phi}+\ddot{\theta})+(\dot{\psi}+\dot{\theta}+\dot{\phi}) \frac{1}{2}[\dot{\psi}-\dot{\phi}-\dot{\theta}]-\frac{1}{2}\left[\dot{\phi}^{2}+\dot{\psi}^{2}+\dot{\theta}^{2}\right] \\
G^{3}{ }_{3} & =-(\ddot{\psi}+\ddot{\phi})+(\dot{\psi}+\dot{\theta}+\dot{\phi}) \frac{1}{2}[\dot{\theta}-\dot{\phi}-\dot{\psi}]-\frac{1}{2}\left[\dot{\phi}^{2}+\dot{\psi}^{2}+\dot{\theta}^{2}\right]
\end{aligned}
$$

and

$$
G^{0}{ }_{0}=-\frac{1}{2}[\dot{\phi}+\dot{\psi}+\dot{\theta}]^{2}+\frac{1}{2}\left[\dot{\phi}^{2}+\dot{\psi}^{2}+\dot{\theta}^{2}\right]
$$

The comoving coordinate used in this computation and the spin density moving transport with the fluid, with velocity $v^{j}$ leads to the followng relations for the energy-stress tensor

$$
T_{i j}=(\rho+p) v_{i} v_{j}-p g_{i j}
$$


and the constraint on the torsion and spin density as

$$
S_{i j}^{k}=S_{i j} v^{k}
$$

where the Weyssenhoff-Raabe condition is as usual given by

$$
S_{i j}{ }^{j}=S_{i j} v^{j}=0
$$

we assumed that, to simplify computations, the spins are polarised along the $x$-axis so that only the component $S_{23}=-S_{32}$ is non-vanishing. One of the EC equations is

$$
8 \pi S_{i j}{ }^{k}=T_{i j}{ }^{k}
$$

where $\mathrm{T}$ is the torsion tensor. The EC equations are

$$
G_{0}^{0}-64 \pi^{2} S^{2}=-8 \pi T_{0}^{0}
$$

where $S^{2}=S_{23} S^{23}$. The remaining equations are

$$
G_{A}^{A}-64 \pi^{2} S^{2}=-8 \pi T_{A}^{A}
$$

where here $(A=1,2,3)$, however, the index A does not obey the Einstein $\mathrm{s}$ summation convention. The final equation is

$$
8 \pi T^{23}=e^{2 \theta}(\dot{\psi}-\dot{\theta})
$$

This last equation yields the metric constraint

$$
\dot{\psi}=\dot{\theta}
$$

and the remaining equations yields

$$
\dot{\phi}-\dot{\psi}=3 \alpha R^{-3}
$$

where the parameter $\alpha$ is a constant related to shear. This, by the way is an important ingredient to neutrino cosmology [12]. In the last formula for $\mathrm{R}$ is given by

$$
R^{3}=\exp [\phi+\psi+\theta]
$$

From the Bianchi identities one obtains

$$
\partial_{t}\left(\rho R^{3}\right)=16 \pi S \partial_{t}\left(S R^{3}\right)
$$


According to Raychaudhuri a simple solution to these EC equations yields

$$
\rho=\rho_{1} V^{-1}+\rho_{2} V^{-\alpha}
$$

and for spin density

$$
S^{2}=C^{1}{ }_{1} V^{-2}+C^{2}{ }_{2} V^{-\alpha}
$$

where $\mathrm{C}$ s represent constants.Here $V=R^{3}$ is the metric volume. Assuming that the spins alignments remain frozen, we obtain

$$
\rho R^{3}=\frac{3}{4 \pi} M
$$

and

$$
S^{2} R^{6}=\text { const }=k
$$

which shows that the overall spin density squared decays with the universe expansion as

$$
S^{2}=\frac{k}{R^{6}}
$$

which explain clearly why after inflation when the radius of the universe grows very fast the torsion asssociated to non-dynamical torsion, Cartan torsion decays very fast with expansion. In what follows, we shall present the idea pf chiral metric from the EC solution. To simplify the computations we shall assume that, in these anisotropic Einstein-Cartan universe due to the presence of torsion and neutrinos, the metric function $\psi$ and $\theta$ coincides. This satisfies the above constraints however is a non-necessary condition for the EC solution, and therefore is considered a particular exact solution of EC anisotropic cosmology. Let us now consider the substitution of the metric function constraint into the Einstein-Cartan equations. Hence the $\mathrm{EC}$ equations reduce to two differential equations of the remaining metric functions

$$
2 \ddot{\psi}+\frac{5}{8} \dot{\psi}^{2}=-\frac{n_{\nu}^{2} h^{2}}{16}
$$

and the constraint

$$
\dot{\phi}=\dot{\psi}+3 \alpha R^{-3}
$$

where here we consider that the energy density which creates curvature and torsion are neutrino density. To solve these equations let us use a simplified ansatz for the $\psi=e^{\Lambda t}$. Substitution of this expression one obtains

$$
\ddot{\psi}=\Lambda \dot{\psi}
$$


Thus a final substitution yields

$$
5 x^{2}+8 \Lambda x+c=0
$$

where $x=\dot{\psi}$ and $c=-n_{\nu}{ }^{2} h^{2}$. This reduces the last differential equation to a simple algebraic second order equation, which solution is

$$
\dot{\psi}_{ \pm}=-8 \Lambda \pm \sqrt{10} n_{\nu} h
$$

the other differential equation reduces to

$$
\dot{\phi}_{ \pm}=3 \alpha R^{-3} \pm \sqrt{10} n_{\nu} h
$$

Therefore these two last expressions are easily solved exactly to yield the chiral metric solutions

$$
\psi_{ \pm}=\left[-8 \Lambda \pm \sqrt{10} n_{\nu} h\right] t
$$

the other is a little more involved, since in

$$
\phi_{ \pm}=3 \alpha \int R^{-3} d t \pm \sqrt{10} n_{\nu} h t
$$

Thus the chiral metric functions solution is

$$
g_{22}=g_{33}=\exp \left[2 \psi_{ \pm}\right]=\exp \left[-8 \Lambda \pm \sqrt{10} n_{\nu} h\right] t
$$

the other is a little more involved, since in

$$
g_{11}=\exp \left[2 \phi_{ \pm}\right]=\exp \left[3 \alpha \int R^{-3} d t \pm \sqrt{10} n_{\nu} h t\right]
$$

Note that the idea of chiral metric here is quite diffent from torsion, since in the chiral torsion case as we shall show in next section, does not affect the overall line element geometry in terms of sign but certainly affects the metric components which may decay depending on the sign of the neutrino helicity. This can be seen by considering the relation between torsion and the spin, of neutrinos, which in high energy astrophysics remains the same. Note that if the neutrinos are left handed, the plus-minus sign shoub be chosen negative and from the last two expressions the chiral metric functions vanish, therefore the Bianchi type I metric is resuced to the flat Riemannian metric, far away from neutrino sources. 


\section{Neutrino Helicity Flip from Axial Anomaly with Torsion}

Let us now address the problem of checking how the cosmic neutrinos are affected by torsion and axial anomaly together. It means we want to investigate how neutrinos flip from the present of torsion and axial anomaly with magnetic and electric fields backgrounds. Recently we have investigated the effect of torsion and cosmological metric [6] and presented this expression, here addapted in principle to the anisotropic metric as

$$
\frac{1}{R^{3}} \partial_{t}\left(R^{3} n_{5}\right)+S_{0} n_{5}=2 \frac{\alpha}{\pi}(E . B)
$$

where $n_{5}=n_{5}{ }^{L}-n_{5}{ }^{R}$ where $\mathrm{L}$ and $\mathrm{R}$ indices represent the right and left fermion handness. Before we apply this expression to the Bianchi type I metric, we shall address the much simpler problem of De Sitter spacetime so important in quantum gravity. In this case $R(t)=R_{0} \exp [\Lambda t]$ and the chiral equation reads

$$
\frac{\dot{R}}{R}+\frac{\dot{n_{5}}}{3 n_{5}}=\frac{1}{3}\left[\frac{2 \alpha_{e m}}{\pi h}-S_{0}\right](\mathbf{E . B})
$$

This equation implies that

$$
\frac{d \ln n_{5}}{d t}+\frac{d \ln R}{d t}=\left[\frac{2 \alpha_{e m}}{\pi h}-S_{0}\right](\mathbf{E} . \mathbf{B})
$$

In this better form one can easily obtain the solution

$$
n_{5}=n_{5}{ }^{0} \exp \left[\left[\frac{2 \alpha_{e m}}{\pi h}-S_{0}\right] \int(\mathbf{E} . \mathbf{B}) d t\right] \exp [-\Lambda t]
$$

To analyse the physics of this solution we first examine the second exponential which is a decay if the cosmological constant is positive. It means that this term contributes to the decay of the left-handed neutrino specie. Of course this is valid when the torsion vanishes. Let us examine the role chiral torsion would play, in the Bianchi type I in EC cosmology. This implies that the axial anomaly equation. The main difference is that the axial anomaly term E.B , which now in this curved torsionful spacetime reads

$$
\frac{d \ln \left(R^{3} n_{5}\right)}{d t}=\frac{1}{R^{3}}\left[\frac{2 \alpha_{e m}}{\pi h}-S_{0}\right](\mathbf{E} . \mathbf{B})
$$


where now we choose the axial anomaly factor as

$$
\mathbf{E . B}=\left[c_{1} \exp (-2 \psi)+c_{2} \exp (-2 \theta)\right]
$$

where

$$
c_{A}=E_{A} \cdot B_{A}
$$

From these expressions, the axial anomaly equation in Bianchi type I EC cosmology reads

$$
\left.\frac{d \ln \left(R^{3} n_{5}^{ \pm}\right)}{d t}=\left[\frac{2 \alpha_{e m}}{\pi h}-S_{0}\right]\left(c_{1}+c_{2}\right) \pm \sqrt{10} n_{\nu} h\right]
$$

which solution is

$$
n_{5}{ }^{ \pm}=n_{5}^{0} \exp \left[ \pm\left[\sqrt{10} n_{\nu} h-S_{0}\left(c_{1}+c_{2}\right)\right] t\right]
$$

From this solution we see that the chiral torsion contribution is

$$
n_{5}{ }^{ \pm}(\text {torsion }) \sim \exp \left[\mp\left[S_{0}\left(c_{1}+c_{2}\right)\right] t\right]
$$

This shows that the left-handed neutrinos are favoured by right-chiral torsion, in Bianchi type I EC cosmology, as we wish to prove. Let us now address the issue of chiral flipping equation

$$
\partial_{t} \mu_{5}+\mathcal{O}\left(\nabla \mu_{5}\right)=\frac{2 \alpha_{e m}}{\pi}(\mathbf{E . B})
$$

To simplify the computations here, we assume that the second term on the LHS of last equation may be dropped in chiral cosmology. Therefore, in the presence of chiral torsion the axial anomaly equation reads

$$
\partial_{t} \mu_{5}+\Gamma_{f l i p}^{\text {eff }} \mu_{5}=\frac{2 \alpha_{e m}}{\pi}(\mathbf{E . B})
$$

From the expressions for E.B and the $\psi$ above one obtains

$$
\partial_{t} \mu_{5}+\Gamma_{f l i p} \text { eff }_{\mu_{5}}=\frac{2 \alpha_{e m}}{\pi} c_{1} e^{-2 \psi_{ \pm}}
$$

where by definition, the effective chiral flip in terms of torsion is

$$
\Gamma_{f l i p}{ }^{e f f}=\Gamma_{5}-S_{0}
$$


which shows that a chiral right-hand torsion, where $S_{0}>0$ the 0 -component of torsion, atenuates the chiral flip. This differential ordinary equation maybe solved by the sum of a general solution of the homogeneous equation and a particular solution of the non-homogeneous. The sum of these two soltions are

$$
\mu_{5}=c_{3} \mu_{51}+c_{4} \mu_{52}
$$

where $c_{3}$ and $c_{4}$ are integration constants. These ODEs are

$$
\partial_{t} \mu_{51}=\frac{2 \alpha_{e m}}{\pi} c_{1} e^{m p \sqrt{10} n_{\nu} t}
$$

where we define the vanishing of the effective flip rate $\Gamma_{\text {flip }}$ eff yields a constraint between torsion and chiral flipping rate $S_{0}=\Gamma_{f l i p}$ and the particular solution is

$$
\mu_{51}=\frac{2 \alpha_{e m}}{m p \sqrt{10} n_{\nu} \pi} c_{1} e^{m p \sqrt{10} n_{\nu} t}
$$

where the term of the RHS of the equation results in the integration of the exponent. Now let us solve the equation

$$
\partial_{t} \mu_{52}+\Gamma_{f l i p}{ }^{e f f} \mu_{52}=0
$$

which yields the solution

$$
\mu_{52}=\mu_{50} \exp \left[\Gamma_{f l i p}{ }^{e f f} t\right]
$$

Note that in this second solution when chiral torsion 0-component is $S_{0} \leq$ $\Gamma_{\text {flip }}, 3$ this solution decays in time as the universe expands. In case of $\mu_{5}$ one notes that this piece of the chiral chemical potential survives for left-hand chiral neutrinos, which is the actual universe observational astrophysical result in the case of the SM standard model of astroparticle physics.

\section{Conclusions}

In this paper we have investigate the chiral flipping of neutrinos in the presence of chiral torsion in the Bianchi type I anisotropic cosmology. Results in neutrino asymmentries and the handness of chiral torsion seems to be inverse, meaning that when the chiral torsion is right-handed the neutrinos are 
left-handed as happens in the observable universe predicted by the SM of particle physics It is suggested that by making use of an oscillating Bianchi type IX metrics [?] with torsion and shear, maybe used to induce neutrino oscillations in this background. This work is now in progress.

\section{Acknowledgements}

I would like to my gratitude to P Pavlovic, F W Hehl and C Sivaram for discussions on subject of this paper. Thanks are due to my wife Ana Paula Teixeira Araujo for her full support and patience during the time this paper was carried out. Financial support from University of State of Rio de Janeiro (UERJ) is grateful acknowledged.

\section{References}

[1] D Palle, On chirality of vorticity of the Universe, Entropy, 1-8 (2012).

[2] V de Sabbata and C Sivaram, Spin and Torsion in Gravitation, world scientific, New York, (1994). V N Ponomarev, A O Barvinsky and Yuri Obukhov, Gauge approach and Quantization Methods in Gravity Theory, NAUKA, Moscow, (2017). F W Hehl and Yu N Obukhov, Foundations of Electrodynamics: Charge Flux and Metric, Birkhauser (2003) Prog in Math Phys. L C Garcia de Andrade, Cosmic magnetism in modified theories of gravity, (2017) Ed European Universitaires.

[3] J Magueijo, T Zlosnik and S Speziale, Phys Rev D 102, 064006 (2020).

[4] C Karananas, Eur Phys J C (2019)

[5] B P Dolan, Class Quantum Gravity 27 : 9 (2010) 095010.

[6] L Garcia de Andrade, Eur Phys J C 78: 254 (2018) and Eur Phys J C 77: 401 (2017). L C Garcia de Andrade, Eur Phys J C 78: 530 (2018).

[7] L C Garcia de Andrade, Class Quantum Gravity 38 (2021). L C Garcia de Andrade, Gravitational waves LIGO data induced from spin-0 and spin-1 contortion propagating modes in teleparallelism, (2020) Eur Phys J Plus 136, 146 (2021). P Mahato, Mod Phys Lett A 17, 1991 (2002). 
[8] A K Raychaudhuri, Theoretical Cosmology, Oxford studies in physics, Clarendon Press, Oxford. C Lammerzahl, Phys Lett A 228 (1997) 223231.

[9] V de Sabbata and M Gasperini, Neutrino oscillation in the presence of torsion, Il Nuovo Cimento A 65, 479, (1981).

[10] S Capozziello, G Iovane, G Lambiase, C Stornaiolo, Fermion Helicity Flip Induced by Torsion Field, Europhys Letters (1999). A Kostelecky, N Russell and J D Tasson, Phys Rev Lett 100 (2008) 111102. H T Nieh, Torsion and Gauge Theory, arXiv: 1712.00901v 2 [gr-qc] june 2018.

[11] A Boyarsky, J Froelich, O Ruchayski, Phys Rev Lett (2010).

[12] J Lesgourgues, G Mangano, G Miele and S Pastor, Neutrino Cosmology, Cambridge University Press (2013). S Yajima, Class Quantum Grav 13,2423 (1996). H T Nieh, Int J Mod Phys A 22,5237 (2007).

[13] L Garcia de Andrade and G Monerat, Bianchi type IX world with torsion and shear, arXives: (2002) 